\title{
Stopping: from Peripheral to Central Nuclear Collisions at the SPS
}

\author{
G.E. Cooper ${ }^{\mathrm{a}}$ for the NA49 Collaboration \\ ${ }^{a}$ Lawrence Berkeley National Laboratory, University of California, Berkeley, USA.
}

New results on baryon stopping at the CERN SPS, measured by the inclusive net proton $(\mathrm{p}-\overline{\mathrm{p}})$ rapidity distribution, are presented for centrality selected $\mathrm{Pb}+\mathrm{Pb}$ and $\mathrm{p}+\mathrm{Pb}$, as well as minimum bias $p+p$ collisions. A smooth evolution in the degree of stopping is observed when going from small to large systems, with the greatest stopping in the most central $\mathrm{p}+\mathrm{Pb}$ collisions. On the other hand, longitudinal distributions of produced particles vary little with the size of the system, and the total yield of pions scales approximately with the number of participating nucleons.

\section{Introduction}

In the early phase of a nuclear collision, participating nucleons convert a large fraction of their kinetic energy into other degrees of freedom. How this occurs sets the baryon density created in the collision and determines the energy available for particle production and expansion. At the CERN SPS, baryon stopping in collisions using light nuclear projectiles (NA35 [1]) and in central $\mathrm{Pb}+\mathrm{Pb}$ collisions ( $\mathrm{NA} 49$ [2]) were studied previously. The results presented here extend these studies over nearly the full range of nuclear system size.

\section{Experimental Details}

The NA49 experiment is described in detail in [3]. Charged particles emitted from a collision are detected over a large fraction of phase space by tracking in four large volume time projection chambers (TPC's). Particle momentum (p) and velocity are determined from tracking through a magnetic field and measuring the specific energy loss in the TPC gas $(d E / d x)$. Individual primary particle spectra are obtained by unfolding the measured $d E / d x$ distributions in $\mathrm{p}$ bins with a model of the TPC response and correcting for feeddown from significant decay channels. An estimate of the systematic errors can be found in $[4]$.

Centrality samples are selected in the case of $\mathrm{Pb}+\mathrm{Pb}$ collisions by a zero-degree calorimeter, which accepts charged and neutral particles emitted within $0.3^{\circ}$ of the beam. The zero-degree energy measurement is well correlated with produced transverse energy and multiplicity, and provides a measurement of collision impact parameter with a relative resolution of $15 \%[5,6]$. Collisions are divided into six centrality bins using the zero-degree energy alone [4].

In each centrality bin, a direct estimate of the number of interacting or participating nucleons $\left(\mathrm{N}_{\text {part }}\right)$ is made by estimating the net baryon number carried by produced 

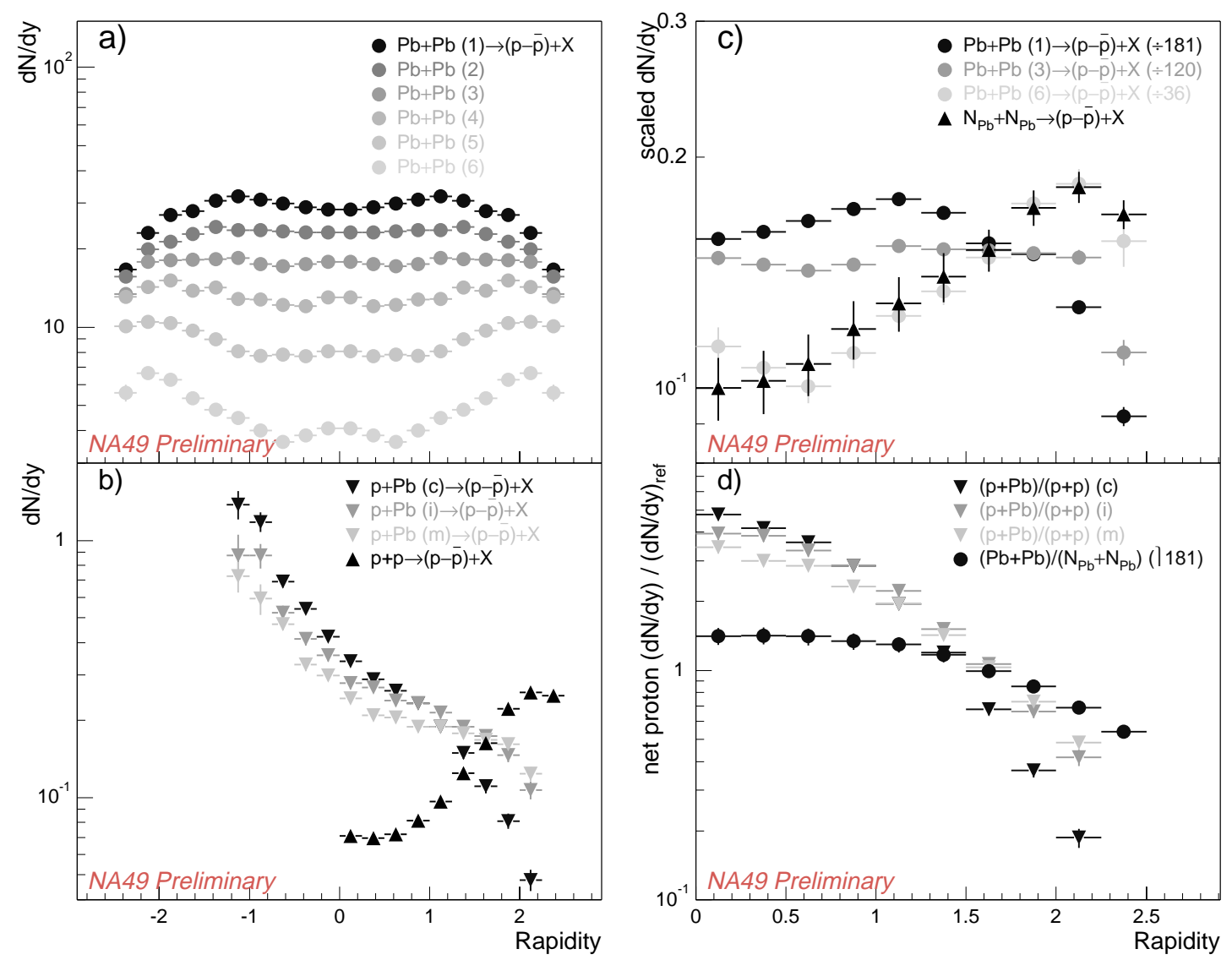

Figure 1. Rapidity distributions of net protons $(p-\bar{p})$ for various nuclear systems. Panel a) shows results for peripheral (6) to central (1) $\mathrm{Pb}+\mathrm{Pb}$ collisions. Panel b) shows results for minimum bias $(\mathrm{m})$, intermediate (i), and central (c) $\mathrm{p}+\mathrm{Pb}$ collisions. Panel c) shows three of the $\mathrm{Pb}+\mathrm{Pb}$ centrality bins, normalized by the estimate of the mean number of participating nucleons for that bin, along with the effective nucleon-nucleon reaction which is appropriate for comparison to $\mathrm{Pb}+\mathrm{Pb}$. Panel d) shows central $\mathrm{Pb}+\mathrm{Pb}$ and $\mathrm{p}+\mathrm{Pb}$ results, normalized by the appropriate reference collision.

particles. Since measurement of the charged particle spectra covers nearly all of phase space forward of the center of mass of the system, little extrapolation is required. Net baryon number is carried by the net protons, net neutrons $(n-\bar{n})$, and net hyperons $(Y-\bar{Y})$. The number of net neutrons produced in the collision is assumed to be proportional to the number of net protons. The total number of net hyperons (including multi-strange baryons) is estimated by balancing the net strangeness carried by the strange mesons. The net strangeness carried by both the charged and neutral kaons is assumed to be twice that of the net charged kaons $\left(\mathrm{K}^{+}-\mathrm{K}^{-}\right)$. Consequently,

$\mathrm{N}_{\text {part }}=(1+\alpha)(\mathrm{p}-\overline{\mathrm{p}})+2 \frac{1+\beta}{1+2 \beta}\left(\mathrm{K}^{+-} \mathrm{K}^{-}\right)$, 


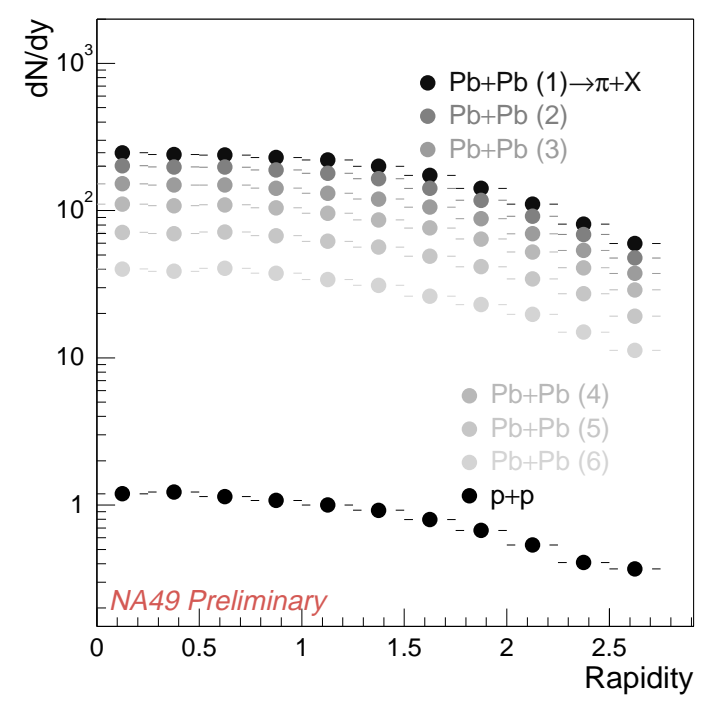

Figure 2. Rapidity distributions of $\left(\pi^{+}+\pi^{-}\right) / 2$ for $p+p$ and centrality selected $\mathrm{Pb}+\mathrm{Pb}$ collisions.

\begin{tabular}{ccccc}
\hline bin & $\begin{array}{c}\text { frac. of } \\
\text { cross sec. }\end{array}$ & $\left\langle\mathrm{N}_{\text {part }}\right\rangle$ & $\begin{array}{c}\mathrm{b}(\mathrm{fm}) \\
(\mathrm{a})\end{array}$ & $\begin{array}{c}\mathrm{b}(\mathrm{fm}) \\
(\mathrm{b})\end{array}$ \\
\hline 1 & $0-0.05$ & $362 \pm 12$ & 2.2 & 2.2 \\
2 & $0.05-0.14$ & $304 \pm 16$ & 4.6 & 4.0 \\
3 & $0.14-0.23$ & $241 \pm 16$ & 6.5 & 5.6 \\
4 & $0.23-0.31$ & $188 \pm 16$ & 8.0 & 6.8 \\
5 & $0.31-0.48$ & $130 \pm 14$ & 9.5 & 8.3 \\
6 & $0.48-1.00$ & $72 \pm 8$ & 11.5 & 10.0 \\
\hline
\end{tabular}

Table $1 . \mathrm{Pb}+\mathrm{Pb}$ centrality bins from most central (1) to peripheral (6). Columns show the covered range in fraction of the total cross section, the mean number of participating nucleons (see text), and the mean impact parameter for the corresponding range in cross section (a), and for which a Glauber calculation gives that number of wounded nucleons (b).

where $\alpha=(\mathrm{n}-\overline{\mathrm{n}}) /(\mathrm{p}-\overline{\mathrm{p}}) \approx 1.07$ and $\beta=(\mathrm{Y}-\overline{\mathrm{Y}})_{[s=2]} /(\mathrm{Y}-\overline{\mathrm{Y}})_{[s=1]} \approx 0.1$ (net $\Omega$ are ignored). These ratios were estimated by averaging the results of several models and measured results. They were assumed to be independent of centrality but using the actual centrality dependence of the ratios would change the results little. The estimated mean number of participating nucleons in each centrality bin is given in table 1 . These estimates include the small extrapolation mentioned above, which ranges from 6 percent for centrality bin 1 to 22 percent for centrality bin 6 . The quoted errors are statistical only. Table 1 shows the range in the $\mathrm{Pb}+\mathrm{Pb}$ interaction cross section of each centrality bin, which was calculated from the measured zero-degree energy spectrum, and the mean impact parameter calculated for this range in cross section from geometry. Also shown is the impact parameter value for which a Glauber calculation yields a number of wounded nucleons equal to the number of participating nucleons estimated from the spectra (used in [7]). Note that the data in bin 5 and especially bin 6 are significantly biased by the experimental trigger.

In the case of $\mathrm{p}+\mathrm{Pb}$ collisions, centrality is estimated by measuring the number of "grey" particles produced by the intra-nuclear cascade in the target nucleus, and is described in [4]. Results are presented for minimum bias, and selected intermediate and central collision samples.

\section{Results}

Figure 1a shows the net proton rapidity distribution per event averaged over events in each of the six $\mathrm{Pb}+\mathrm{Pb}$ centrality samples. In going from peripheral to central collisions, 
the forward yield of net protons is strongly suppressed relative to that at mid-rapidity and the rapidity at which the largest yield is observed moves toward mid-rapidity, indicating a significant increase in stopping from peripheral to central collisions. Figure $1 \mathrm{~b}$ shows the same distributions for $\mathrm{p}+\mathrm{p}$ and $\mathrm{p}+\mathrm{Pb}$. In $\mathrm{p}+\mathrm{Pb}$, the strong enhancement of the yield in the target hemisphere comes from stopping of the target nucleons. Sufficiently forward of mid-rapidity, where this effect is suppressed, the probability of finding a forward going proton decreases strongly in going from $\mathrm{p}+\mathrm{p}$ to $\mathrm{p}+\mathrm{Pb}$ collisions, with the greatest stopping in the central $\mathrm{p}+\mathrm{Pb}$ sample.

To compare $\mathrm{Pb}+\mathrm{Pb}$ to $\mathrm{p}+\mathrm{p}$ directly, one must account for the enhanced yield in $\mathrm{Pb}+\mathrm{Pb}$ of net protons which comes from stopping of neutrons. This is done by using data on $\mathrm{p}+\mathrm{p} \rightarrow \mathrm{n}+\mathrm{X}[8]$, making the simplifying assumption that nucleon stopping is similar in $\mathrm{p}+\mathrm{p}$ and $\mathrm{p}+\mathrm{n}$, and taking an isospin average $(60 \% \mathrm{n}, 40 \% \mathrm{p})$ to construct the effective nucleon-nucleon system $\left(\mathrm{N}_{\mathrm{Pb}}+\mathrm{N}_{\mathrm{Pb}}\right)$ appropriate for comparison to $\mathrm{Pb}+\mathrm{Pb}$. Figure $1 \mathrm{c}$ shows three of the six $\mathrm{Pb}+\mathrm{Pb}$ centrality bins, normalized by the estimated number of participating nucleons, compared to $\mathrm{N}_{\mathrm{Pb}}+\mathrm{N}_{\mathrm{Pb}}$. Figure $1 \mathrm{~d}$ shows a comparison of $\mathrm{Pb}+\mathrm{Pb}$ and $\mathrm{p}+\mathrm{Pb}$, where each has been normalized by the corresponding reference distribution $\left(\mathrm{N}_{\mathrm{Pb}}+\mathrm{N}_{\mathrm{Pb}}\right.$ for $\mathrm{Pb}+\mathrm{Pb}, \mathrm{p}+\mathrm{p}$ for $\left.\mathrm{p}+\mathrm{Pb}\right)$.

Figure 2 show the rapidity distribution of charged pions (mean of $\pi^{+}$and $\pi^{-}$) for $p+p$ and $\mathrm{Pb}+\mathrm{Pb}$. In contrast to the net protons, the shape of these distributions show little change with system size. This is also the case for other produced particles $\left(\mathrm{K}^{+}, \mathrm{K}^{-}\right.$, and $\bar{p}$, data not shown). Additionally, it is shown in [4] that the yield of pions increases smoothly with, and only slightly faster than, the increase in the number of participating nucleons. Thus, the increase in stopping observed in going from small to large systems is not reflected in the longitudinal distributions of produced particles or in the yield of pions.

As shown in [4] and [9], both the mean transverse momentum of produced particles heavier than pions and the yield of strange particles increases with increasing centrality. The question remains whether this increase can be understood merely in terms of the increased stopping or whether additional collective effects arise during expansion of the larger system from more central collisions.

\section{REFERENCES}

1. T. Alber et al. [NA35 Collaboration], Eur. Phys. J. C 2 (1998) 643.

2. H. Appelshäuser et al. [NA49 Collaboration], Phys. Rev. Lett. 82 (1999) 2471.

3. S. Afanasiev et al. [NA49 Collaboration], Nucl. Instrum. Methods Phys. Res., Sect. A 430 (1999) 210.

4. F. Sikler [NA49 Collaboration], in these proceedings.

5. T. Alber et al. [NA49 Collaboration], Phys. Rev. Lett. 75 (1995) 3814.

6. H. Appelshäuser et al. [NA49 Collaboration], Eur. Phys. J. A 2 (1998) 383.

7. A.M. Poskanzer [NA49 Collaboration], in these proceedings.

8. J. Engler et al., Nucl. Phys. B 84 (1975) 70.

9. C. Höhne [NA49 Collaboration], in these proceedings. 